\title{
Lateral Motion and Departure of Vapor Bubbles in Nucleate Pool Boiling on Thin Wires in Microgravity
}

\author{
J. F. Zhao*, S. X. Wan, G. Liu, Z. D. Li, Y. H. Lu, N. Yan \\ National Microgravity Laboratory, Institute of Mechanics, Chinese Academy of Sciences. Beijing, \\ 100080, China \\ E-mail: jfzhao@imech.ac.cn
}

\begin{abstract}
A space experiment on bubble behavior and heat transfer in subcooled pool boiling phenomenon has been performed utilizing the temperature-controlled pool boiling (TCPB) device both in normal gravity in the laboratory and in microgravity aboard the $22^{\text {nd }}$ Chinese recoverable satellite. The fluid is R113 at $0.1 \mathrm{MPa}$ and subcooled by $26^{\circ} \mathrm{C}$ nominally. A thin platinum wire of $60 \mu \mathrm{m}$ in diameter and $30 \mathrm{~mm}$ in length is simultaneously used as heater and thermometer. Only the lateral motion and the departure of discrete vapor bubbles in nucleate pool boiling are reported and analyzed in the present paper. A scale analysis on the Marangoni convection surrounding a bubble in the process of subcooled nucleate pool boiling leads to formulas of the characteristic velocity of the lateral motion and its observability. The predictions consist with the experimental observations. Considering the Marangoni effect, a new qualitative model is proposed to reveal the mechanism underlying the bubble departure processes and a quantitative agreement can also be acquired.
\end{abstract}

Key words: microgravity, nucleate pool boiling, lateral motion, bubble departure diameter, Marangoni effect

\section{INTRODUCTION}

Pool boiling in microgravity has become an increasing significant subject for investigation, since many potential applications exist in space and on planetary neighbors due to its high efficiency. However, the investigation in microgravity suffers for unique and stringent constraints in terms of size, power and weight of experimental apparatuses, and of number and duration of the experiments. Thus, only a partial and in some aspects contradictory knowledge of microgravity boiling has been attained so far. On the progress in this field, several comprehensive reviews are available. They are authored by Straub[1], and Di Marco \& Grassi[2], among many others.

Boiling is a very complex and illusive process because of the interrelation of numerous factors and effects as the nucleate process, the growth of the bubbles, the interaction between the heater's surface with liquid and vapor, the evaporation process at the liquid-vapor interface, and the transport process of vapor and hot liquid away from the heater's surface. For a variety of reasons, fewer studies have focused on the physics of the boiling process than have been tailored to fit the needs of engineering endeavors. As a result, the literature has been flooded with the correlations involving several adjustable, empirical parameters. These correlations can provide quick input to design, performance. and safety issues and hence are attractive on a short-term basis. However, the usefulness of the correlations diminishes very quickly as parameters of interest start to fall outside the range of physical parameters for which the correlations were developed. Thus, the physics of the boiling process itself is not properly understood yet, and is poorly represented in the most correlations, despite of almost seven decades of boiling research.

The present work is a research effort on subcooled pool boiling heat transfer both in normal gravity on Earth and in microgravity aboard the $22^{\text {nd }}$ Chinese recoverable satellite. Only the lateral motion and the departure of discrete vapor bubbles in nucleate pool boiling are reported and analyzed in the present paper. 


\section{EXPERIMENTAL FACILITY}

A space experiment was conducted by our group aboard the $22^{\text {nd }}$ recovery satellite of China in September, 2005. The level of residual gravity is estimated as $10^{-3} \sim 10^{-5} g_{0}$. In order to perform such studies in normal gravity on Earth as while as in microgravity both in the Drop Tower Beijing of NMLC (National Microgravity Laboratory/CAS) and aboard a Chinese recovery satellite, a TemperatureControlled Pool Boiling (TCPB) device has been developed. Detailed description of the experimental facility can be read in Wan et al. ${ }^{[3]}$ Therefore, only a simple description will be presented briefly here. A platinum wire of $60 \mu \mathrm{m}$ in diameter and $30 \mathrm{~mm}$ in length is simultaneously used as heaters and thermometers, with the advantage that because of its low thermal capacity, it reacted almost without any delay on changes in temperature and heat transfer, respectively. The ends of the wire are soldered with copper poles of $3 \mathrm{~mm}$ in diameter to provide a firm support for the wire heater and low resistance paths for the electric current. The heater resistance, and thus the heater temperature, is kept constant by a feedback circuit similar to that used in constant-temperature hot-wire anemometry. Its adjustment is controlled by varying the resistance of the changeable resistance network. This resistance network comprises 16 parallel resistors and a 16-channel analog switch, which is controlled by a SCM (single-chip-microcomputer). Thus, 16 set-points of heater temperature can be obtained. An "up-down-up" procedure is used for the heater temperature. According to Straub [1], each state lasts about 30 seconds in order to obtain steady pool boiling.

The boiling chamber is filled with about $700 \mathrm{ml}$ of degassed R113, and fixed inside an air-proof container. A bellows connected with the chamber will allow the pressure in the chamber to be approximately constant. Two platinum resistance thermometers with a range of $0 \sim 60^{\circ} \mathrm{C}$ are used to measure the bulk temperature of the fluid in the boiling chamber, which are calibrated to within $0.25^{\circ} \mathrm{C}$. The absolute pressure within the boiling chamber is measured using a pressure transducer with a range of $0 \sim 0.2 \mathrm{MPa}$ and an accuracy of $0.25 \% \mathrm{FS}$ (full scale). Two LEDs (light-emitting diode) are used to light the boiling chamber through a diffusion window at the chamber bottom. A CCD video camera is used to obtain images of the motion of vapor bubble or film around the heater, which is recorded by a VCR at a speed of $25 \mathrm{fps}$. The voltages across the heater and a reference resistance, which is used to measure the electric current through the heater, are sampled at a rate of $20 \mathrm{~Hz}$ in space experiment, while a much higher sampling rate of $1000 \mathrm{~Hz}$ is used in the ground experiments before and after the space flight. A lower sampling rate of $1 / 3 \mathrm{~Hz}$ is used for the outputs of the pressure transducer and the platinum resistance thermometers both in space and on the ground.

\section{BUBBLE LATERAL MOTION AND ITS SCALE ANALYSE}

It's observed that there exists a forward-and-backward lateral motion of discrete vapor bubbles along the wire before their departure from the wire in microgravity. Figure 1 shows the typical forwardand-backward lateral motion of a bubble of about $0.9 \mathrm{~mm}$ in diameter. The lateral velocity is about $20 \mathrm{~mm} / \mathrm{s}$, unless it comes in collision with and bounces off an adjacent bubble. Generally, the lateral velocity increases with the decrease of the bubble size. This kind of lateral motion can lead to the lateral coalescence between adjacent bubbles, and then the new bubble detaches due to its surface oscillation. For example, the above bubble coalesces with another bubble when it moves back to nearly its initial position. The following motion after its departure is shown in Figure 2.

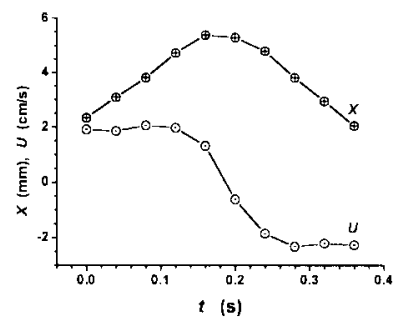

Figure 1: Lateral motion of a typical bubble

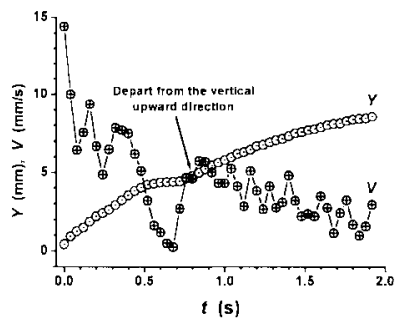

Figure 2: Departure of the former bubble

A hypothesis has been proposed by Zhao et al.[4] that adjacent bubbles entrain each other in thermocapillary or Marangoni flow surrounding them during nucleate boiling of subcooled liquids. The 
entrainment manifests itself as motion of the bubbles toward each other, which promotes their coalescence. A scale analysis on this phenomenon leads to formulas of the characteristic velocity of the lateral motion and its observability as

$U_{0}=\sqrt{\sigma_{T} \Delta T / \rho_{L} d}$

$O b=\frac{U_{0}}{U_{b}}=g^{-1} \sqrt{\frac{\sigma_{T} \Delta T v_{L}^{2}}{\rho_{L} d^{5}}}$

The predictions consist with the experimental observations (Table 1).

Table 1 Bubble lateral motion and its observability

\begin{tabular}{|c|c|c|c|c|c|c|c|}
\hline$\overline{\rho_{L}, \mathrm{~kg} / \mathrm{m}^{3}}$ & $\sigma_{T}, \mathrm{~N} / \mathrm{mK}$ & $\Delta T, \mathrm{~K}$ & $\nu_{L}, \mathrm{~m}^{2} / \mathrm{s}$ & $D, \mathrm{~mm}$ & $U_{0}, \mathrm{~mm} / \mathrm{s}$ & $g / g_{0}$ & $O t$ \\
\hline \multirow{5}{*}{$10^{3}$} & \multirow{5}{*}{$10^{-4}$} & \multirow{5}{*}{10} & \multirow{5}{*}{$10^{-6}$} & 10 & 10 & - & - \\
\hline & & & & & & $10^{-5}$ & $\sim 10^{2.5} \gg 1$ \\
\hline & & & & 1 & 32 & $10^{-2}$ & $\sim 10^{-0.5} \sim 1$ \\
\hline & & & & & & 1 & $\sim 10^{-2.5} \ll 1$ \\
\hline & & & & 0.1 & 100 & 1 & $\sim 1$ \\
\hline
\end{tabular}

\section{BUBBLE DEPARTURE AND ITS MECHANISM}

For the bubble departure phenomanon, three critical bubble diameters, namely $D_{b 1}=0.3 \mathrm{~mm}, D_{b 2}=3.5$ $\mathrm{mm}, D_{b 3}=8.4 \mathrm{~mm}$, are observed in microgravity, which divide the observed vapor bubbles into four regimes with different sizes (Figure 3). Tiny vapor bubbles form and grow on the heater surface unt il their sizes exceed the first critical value, and then depart slowly from the wire. Above the second critical value, however, bubble may stay on the wire, oscillate along the wire, and coalesce with adjacent bubbles, till its size exceeds the third critical value and it will depart from the wire again. The behaviors of tiny bubbles are observed both in microgravity and in normal gravity, while the last two kinds of bubble behaviors are observed only in microgravity aboard the satellite. Presently, no model can predict the whole observation.

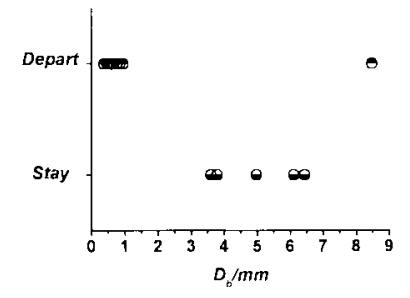

Figure 3: Bubble departure in microgravity

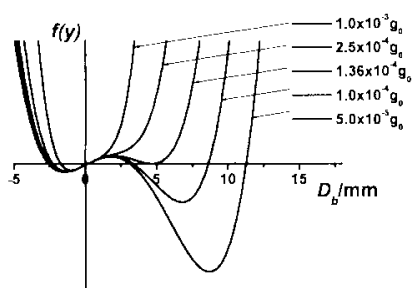

Figure 4: Total force acting on the growing bubble

In order to reveal the mechanism underlying the bubble departure processes, a qualitative model is proposed based on the model of Lee[5] and considering the Marangoni effect. The resulting formula to describe the total force acting on the growing bubble is as

$f(y)=C_{4} y^{4}+C_{3} y^{3}+C_{1} y+C_{0}$

where,

$\mathrm{y}=\tau^{1 / 2}$

$C_{4}=\frac{4}{3} \pi E^{3}\left(\rho_{L}-\rho_{V}\right) g$ 


$$
\begin{aligned}
& C_{3}=-2 K \pi\left|\sigma_{T}\right| T_{1} E^{2} \\
& C_{1}=4 \sigma R_{0} \sin ^{2} \beta+\frac{\pi}{3} \rho_{L} E^{4} \\
& C_{0}=R_{0} E^{3} \rho_{L} \sin ^{2} \beta\left(\frac{1}{3}-\frac{3}{8} C_{d}\right)
\end{aligned}
$$

According to Eq. (3), the following conclusion can be obtained: If $f(y)<0$, the departure force is larger than the resistant force, so the bubble will stay on the heater's surfacc; if $f(y)>0$, the departure force is smaller than the resistant force, so the bubble will depart from the heater's surface. Fig. 4 shows the predictions of Eq. (3) in different gravity conditions. It's shown that there is only one solution when the local gravity is larger than some critical value, and three solutions when the local gravity is smaller than the critical value. Considering the experimental conditions, namely $p_{l}=0.1$ $\mathrm{MPa}, \Delta T_{\text {sub }}=24^{\circ} \mathrm{C}, \Delta T_{\text {sat }}=30^{\circ} \mathrm{C}, \beta=5^{\circ}$, and the residual gravity $g=10^{-4} g_{0}$, a quantitative agreement, namely $D_{b 1}=0.11 \mathrm{~mm}, D_{b 2}=3.4 \mathrm{~mm}, D_{b 3}=8.6 \mathrm{~mm}$, can also be obtained with the empirical parameter for Marangoni effect $K=0.035$.

\section{SUMMARY}

A temperature-controlled pool boiling (TCPB) device has been developed to study the bubble bchavior and heat transfer in subcooled pool boiling phenomenon both in normal gravity and in microgravity. The space experiment has been performed aboard the $22^{\text {nd }}$ Chinese recoverable satellite, while the ground experiments have also been conducted before and after the flight.

Distinct bubble behaviors are observed in microgravity, comparing with those in normal gravity. Lateral motions are observed and a scale analysis on the Marangoni convection surrounding a bubble leads to formulas of the characteristic velocity of the lateral motion and its observability. Three critical bubble diameters are observed for the bubble departure in microgravity, which divide the observed vapor bubbles into four regimes with different sizes. Considering the Marangoni effect, a qualitative model is proposed to reveal the mechanism underlying the bubble departure processes, and a quantitative agreement can also be acquired.

\section{ACKNOWLEDGEMENTS}

The present study is supported financially by the Chinese Academy of Sciences under the grant of KJCX2-SW-L05, and the National Natural Science Foundation of China under the grant of 10432060. The authors greatly appreciate Prof. H. Ohta (Kyushu University, Japan) for the fruitful discussion.

\section{REFERENCES}

1. Straub J. Boiling heat transfer and bubble dynamics in microgravity. Adv. Heat Transfer, 2001; 35: $57-172$

2. Di Marco P, Grassi W. Motivation and results of a long-term research on pool boiling heat transfer in low gravity. Int. J. Therm. Sci., 2002; 41(7): 567-585

3. Wan SX, Zhao JF, Liu G, Hu WR. TCPB device: description and preliminary ground experimental results. In: 54th Int. Astronautical Cong., Sep. 29-Oct. 3, 2003, Bremen, Germany

4. Zhao JF, Wan SX, Liu G, Hu WR. Experimental study on subcooled pool boiling in microgravity utilizing Drop Tower Beijing/NMLC. In: Proc. of the $5^{\text {th }}$ Int. Symp. on Multiphase Flow, Heat Mass Transfer \& Energy Conversion, Vol. 4, pp. 1730-1735, Xi'an Jiaotong Univ. Press, Xi'an, China, 2006

5. Lee DJ. Bubble departure radius under microgravity. Chem. Eng. Comm., 1992; 117: 175-189 